\title{
La música dentro de las cortes europeas del siglo XVI. El modelo de Il Cortegiano y el papel de las damas en su consolidación. El ejemplo de las cortes italianas ${ }^{1}$.
}

\author{
Music within sixteenth-century European Courts. The model of Il \\ Cortegiano and the role of the dames in its consolidation. \\ The example of the Italian Courts
}

\section{Jordi Comellas Solé ${ }^{2}$}

Universidad Rey Juan Carlos

\begin{abstract}
Resumen: Durante el siglo XVI las élites nobiliarias tomaron como modelo de comportamiento al gentiluomo descrito por Baldassare de Castiglione en su Cortegiano. Según este ideal, la música jugaba un papel esencial en la formación de príncipes y cortesanos. El soberano humanista debía conjugar la doble faceta de intérprete y patrón. Paralelamente y bajo este prisma, en las cortes italianas de Mantua y Ferrara la figura de la mujer adquirió una relevancia especial en dos ámbitos, por un lado, en el intenso patronazgo musical ejercido por las princesas de las familias gobernantes y por otro, en la importancia de las damas de palacio y profesionales como intérpretes. Todo ello posibilitó el desarrollo de un estilo de canto de gran refinamiento y de un virtuosismo extremo, explicitado en el concerto delle dame de la corte estense de Ferrara de finales del siglo XVI.
\end{abstract}

Palabras clave: Música; gentiluomo; Baldassare de Castiglione; Isabella d'Este; corte de Ferrara; concerto delle dame; canto.

Abstract: Along sixteenth-century, the noble elites took as their model of behaviour the gentiluomo described by Baldassare de Castiglione in his Cortegiano. According to this ideal, music played an essential role in the education of princes and courtiers.

\footnotetext{
${ }^{1}$ Este trabajo se inscribe dentro de las actividades del proyecto DELYRAMUS: Developing Audiences: Music, Luthiers and Mental Health (602109-CREA-1-2018-1-ES-CULT-COOP1), de la Convocatoria Creative Europe - Culture Sub-programme (2014-2020) - 'Support for European Cooperation Projects 2018' 'Smaller scale cooperation projects' (category 1) - Call for proposals EACEA 32/2017.

2 내ttps://orcid.org/0000-0002-9736-522X
}

(C) 2020 Philostrato. Revista de Historia y Arte 
The humanist sovereign had to combine the double side of performer and patron. At the same time and under this prism, woman's role in the Italian Courts of Mantua and Ferrara acquired a special relevance in two areas: on the one hand, in the intense musical patronage exercised by the princesses of the ruling families and, on the other hand, in the importance of noblewomen and professionals as performers. All this made it possible to develop a highly refined and extremely virtuous singing style, depicted on Este's Court concerto delle dame found in Ferrara in late sixteenthcentury.

Keywords: Music; gentiluomo; Baldassare de Castiglione; Isabella d'Este; Ferrara's Court; concerto delle dame; singing.

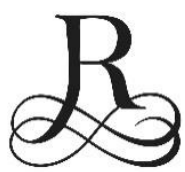

esultaría casi imposible abordar la interacción entre príncipes, nobles y músicos bajo la óptica del modelo de corte en la Italia del siglo XVI sin tomar como referencia al gentiluomo descrito por Baldassare de Castiglione en su Cortegiano. A pesar de la existencia de cortes anteriores al referente urbinés, desde la borgoñona a la aragonesa de Nápoles, será precisamente la de los Montefeltro y las pequeñas cortes padanas como Ferrara y Mantua las que definieron un marco en el que el poder se asentó en la interacción entre el príncipe y los personajes que le rodeaban ${ }^{3}$. El sistema cultural de la corte orbitaba en torno a la relación humana de sus moradores, a su capacidad e ingenio para dominar el arte de la conversación entendido como instrumento de supervivencia y promoción alrededor del soberano4. La regla universal que simbolizaba el centro de la representación del poder expuesta por Castiglione consistía en una huida de la afectación en las relaciones humanas. El cortesano ideal debía esconder esfuerzo y fatiga en su comportamiento y mostrar cierta "naturalidad" en sus actos. A partir de la definición de este proceder surgió el concepto de sprezzatura en cuanto a disimulo de la dificultad como pauta de conducta. La escena de la corte y de las relaciones sociales que se generaron en ella se sucedieron bajo el signo de una teatralidad global que inspiró a los centros de poder europeos desde las primeras décadas del siglo XVI hasta finales del siglo XVIIIs.

El ideal de cortesano se forjó a través de la educación. La educación que moldeó el Humanismo fue personalizada, formadora de élites. Una escuela pensada como Academia, flexible, que se estabilizó y uniformó a finales del siglo XVI con la Ratio Studiorum de los colegios jesuitas'.

La nueva pedagogía tuvo gran arraigo en los pequeños estados italianos desde el siglo XV. La educación entendida como formadora de élites se

\footnotetext{
${ }^{3}$ Cesare Mozzarelli, Antico regime e modernità, (Roma: Bulzoni, 2008), p. 156.

${ }^{4}$ Amedeo Quondam, El discurso cortesano, ed. Eduardo Torres Corominas, (Madrid: Polifemo, 2013), pp. 65-66.

${ }^{5}$ Mozzarelli, Antico regime e modernità, p. 157.

${ }^{6}$ Quondam, El discurso cortesano, pp. 202-203. Para un estudio detallado de los programas de educación jesuitas de finales del siglo XVI ver Stefano Lorenzetti, «"Per animare agli esercizi nobili". Esperienza musicale e identità nobiliare nei collegi di educazione», Quaderni Storici, 32, 2, (1997), pp. 435-460.
} 
encuentra en muchas ciudades con corte, como Mantua. Es en la ciudad de los Gonzaga donde se llevó a cabo uno de los más interesantes experimentos educativos del Humanismo, Ca'Zoiosa, dirigida por el pedagogo Vittorino da Feltre (muerto en 1446). Vittorino, bibliotecario del marqués Gianfrancesco, fundó una escuela inicialmente pensada para formar a los descendientes de la familia Gonzaga pero que al poco tiempo se nutrió con jóvenes, no solo nobles, provenientes de otras partes de Italia y Europa, como el futuro duque de Urbino Federico de Montefeltro7. El programa de estudios de Ca'Zoiosa se organizaba en torno a los autores clásicos y a las artes del trivio (gramática, dialéctica y retórica) y del cuadrivio (matemáticas, geometría, astronomía y música). La filosofía jugaba igualmente un papel importante. El aprendizaje incluía ejercicios corporales propios de la formación guerrera que configuraba la educación integral del moderno cortesano.

No todos los educadores de Ca'Zoiosa fueron humanistas. Conocemos por un alumno de la institución, Francesco Prendilacqua, una relación de profesores especializados empleados por Vittorino. Junto a pintores y gramáticos había músicos, bailarines, cantantes e instrumentistas`.

\section{El cortesano y la música}

Dentro del microcosmos de la corte, tras la figura del príncipe encontramos al cortesano. En una sociedad en que el lugar de la corte pasa a ser el centro de la dimensión política y de la dignidad social, la figura del cortesano se entiende como una especie de consciencia iluminada del príncipe: a la dignitas militar propia del caballero, a lo largo del siglo XV se le añade una gravedad intelectual que define la actividad política y social del gentiluomo'o.

Si una de las principales virtudes del cortesano es la de combinar el ser hombre de armas y de letras, la de ser músico adquirirá una especial relevancia. Así, en el capítulo X del primer libro de la obra de Castiglione, titulado «Cómo al perfeto Cortesano le pertenece ser músico, así en saber cantar y entender el arte, como en tañer diversos instrumentos», el autor aconseja quell:

"Habéis de saber, señores, que nuestro Cortesano, a vueltas de todo lo que he dicho, hará el caso que sea músico; y además de entender el arte y cantar bien por el libro, ha de ser diestro en tañer instrumentos. Porque, si bien lo consideramos, ningún descanso ni remedio hay mayor ni más honesto para las fatigas del cuerpo y

\footnotetext{
7 Quondam, El discurso cortesano, p. 205.

${ }^{8}$ Quondam, p. 206.

9 Iain Fenlon, Music and Patronage in Sixteenth-Century Mantua, Volume 1, (Cambridge: Cambridge University Press, 1980), p. 13.

10 Stefano Lorenzetti, Musica e identità nobiliare nell'Italia del rinascimento: educazione, mentalità, immaginario, (Florencia: L.S. Olschki, 2003), pp. 68-69.

11 En este caso nos referimos a la traducción española de Boscán publicada en Barcelona en 1534: Baldassare Castiglione, El Cortesano, trad. Juan Boscán, (Madrid: Alianza Editorial, 2008).
} 
pasiones del alma que la música, en especial en las cortes de los príncipes, adonde no solamente es buena para desenfadar, mas aun para que con ella sirváis y deis placer a las damas, las cuales de tiernas y de blandas fácilmente se deleitan y se enternecen con ella"'2.

En el texto se pueden encontrar algunas de las razones del porqué de la importancia de la música en el ideal educativo aristocrático, así como algunos de los beneficios de su práctica, puesto que la adquisición de ciertas competencias musicales por parte del cortesano, ya sea poder cantar bien "por el libro" (saber leer música), o poder tañer instrumentos, podían significar un importante factor de promoción de la propia imagen pública así como un elemento básico a la hora de sosegar el espíritu en los momentos de asueto. Décadas más tarde de la publicación del tratado de Castiglione, Stefano Guazzo advertía que: "de esta forma nuestra vida, a modo de instrumentos musicales, así en el tirar como en el frenar las cuerdas, deviene más suave"13.

La relación del gentiluomo ideal con la música debía estar regida por el concepto de sprezzatura. Evitando la afectación y disimulando la dificultad de la interpretación, así como el estudio y fatiga derivados de la práctica musical, se accedía a la gratia de la interpretación ${ }^{14}$. A partir de esta premisa había que establecer qué prácticas musicales e instrumentos eran los más aptos para el hombre de corte. En el capítulo I del segundo libro de El Cortesano, Castiglione pone en labios de Federico Fregoso:

"Muy buena música, respondió miser Federico, me parece cantar diestramente por el libro; mas aun pienso que es mejor cantar con una vihuela. Porque toda la dulzura consiste casi en uno que cante solo, y con mayor atención se nota y se entiende el buen modo y el aire no ocupándose los oídos en más de una voz que si se ocupan en muchas, y allí entonces se juzga más delgadamente un yerro por pequeño que sea, lo que no acaece si muchos cantan, porque el uno ayuda al otro. Mas por lo que yo estoy mejor con el cantar con una vihuela, es por lo que vulgarmente llamamos recitar, el cual da tanta gracia y fuerza a las palabras, que es maravilla. Traen asimismo grande y gentil armonía los instrumentos de tecla, porque tienen las consonancias muy perfectas, y fácilmente se pueden hacer en ellos muchas cosas que a nuestros sentidos nos parecen dulces. No deleitan menos una música de cuatro vihuelas de arco, porque es extrañamente suave y artificiosa. El cantar asienta muy bien en todos estos instrumentos; de los cuales bástele al Cortesano tener noticia, aunque cuanto más ecelente fuere en ellos, tanto mejor será, pero no cure mucho de los que Minerva y

\footnotetext{
12 Castiglione, p. 206.

13 "Insomma la vita nostra, a guisa di stromenti musici, hor col tirare, hor col rallentare le corde, diviene più soave". Stefano Guazzo, La civil conversatione, (Venecia: Salicato, 1577), p. 285.

${ }^{14}$ Lorenzetti, Musica e identità nobiliare, pp. 77-81.
} 
Alcibíades desecharon, porque parece que en cierta manera son ascorrosos"15.

Castiglione se muestra ferviente partidario del canto. Hay que tener en cuenta que a principios del siglo XVI la frottola se encontraba en su máximo apogeo en las pequeñas cortes del norte de Italia16. Frente al poder de la polifonía ejercido en las primeras décadas de siglo por autores transalpinos que copaban los cargos más importantes como maestros de capilla, buena parte de la élite humanística reivindicaba la belleza de la simplicidad de la melodía acompañada, el "cantar con una vihuela" citado17. Sin embargo, a la frescura de la canción acompañada Castiglione contrapone la música producida por "cuatro vihuelas de arco", que claramente refiere a la interpretación de una pieza polifónica interpretada por un conjunto de instrumentos de la misma familia de diferentes tamaños, presumiblemente violas da gamba, capaces de abordar la tesitura de las diferentes voces del tejido polifónico (soprano, alto, tenor y bajo)'8. Pero cuando Juan Boscán en su traducción cita a la vihuela y a las vihuelas de arco, ¿a qué instrumentos en concreto se refiere?

En el original de 1528, Castiglione escribe "cantare alla viola" y "delle quattro viole da arco"'19. En la Italia del momento la palabra viola resulta ambigua ya que podía referirse tanto a instrumentos de cuerda pulsada como la vihuela, la guitarra o el laúd, como a instrumentos de arco tales como la

${ }^{15}$ Castiglione, El cortesano, pp. 176-77.

16 Considerada como la forma de canción profana más extendida en el ambiente elitista de Italia a principios del siglo XVI, la frottola floreció entre 1490 y 1530 al amparo de las cortes del noreste peninsular. Se trataba de composiciones cortas cuyos textos, compuestos en formas fijas, solían ser de temática amorosa. Escritas para cuatro voces, se solía cantar la superior mientras que las otras tres eran ejecutadas por un instrumento, muchas veces tañido por el propio cantante. William F. Prizer, "Performance Practices in the Frottola", Early Music, 3, (2011), p. 227. Nacida en Mantua, este tipo de canción pronto se extendió a Ferrara y Urbino bajo el patronazgo de los príncipes de las casas Gonzaga y Este. Gustav Reese, La música en el Renacimiento, trad. José María Martín Triana, vol. 1, (Madrid: Alianza Editorial, 1995), p. 199.

17 La pulsión polifonía-canción generó controversia entre el mundo culto italiano durante todo el siglo XVI. Una muestra de ello es el debate creado al respecto en la Accademia Fiorentina en 1543 y que enfrentó a partidarios de la polifonía escrita (cuya interpretación requería de ensayos y cuyos resultados artísticos no siempre eran los óptimos a causa de su dificultad técnica) con defensores de la canción acompañada (ligada a la poesía, a la reivindicada lengua toscana, y que gozaba del encanto de la simpleza y de cierto aire improvisado). El debate ha sido profusamente estudiado por Robert Nosow, "The Debate on Song in the Accademia Fiorentina", Early Music History, 21, (2002), pp. 175-221.

18 Uno de los procesos que impulsaron la evolución de la viola da gamba a principios del siglo XVI es precisamente la necesidad de experimentar en un instrumento de diversos tamaños capaz de abarcar los diferentes registros vocales propios de la polifonía. De esta forma empezaron a construirse instrumentos de cuerda frotada capaces de interpretar el registro grave, con un cuerpo o caja más grande que requería de una interpretación por parte del intérprete en vertical, alejada de la horizontalidad propia de las vielas medievales o de la lira da braccio. Jacob A. Mariani, "From Poet's aid to Courtier's pastime: an Examination of the Shift in visual style and sounding function of Italian Viols during the Renaissance", (Degree of Master of Arts, Oregon, University of Oregon, 2014), pp. 26-31. Sobre el desarrollo del conjunto o consort de violas da gamba en la Italia del Cinquecento y de su importancia dentro de la historia del instrumento existe un profundo estudio por parte de Hoffmann: Bettina Hoffmann, "Del concerto alto al concerto basso: accordature delle viole da gamba nell'Italia del Cinquecento", Recercare, 16, (2004), pp. 23-67.

${ }_{19}$ Baldassare Castiglione, Il Libro del Cortegiano con una scelta delle Opere minori, ed. Bruno Maier (Turín: UTET, 1981), p. 213. 
viola da gamba o la lira da braccio ${ }^{20}$. Analizando las principales traducciones contemporáneas a la de 1528 se observa que viola se traduce al castellano por Boscán como vihuela, por Jacques Colin al francés (1537) como luc y por Thomas Horby al inglés (1561) como lute. De la misma forma, viole da arco vienen traducidas como vihuelas de arco, violles a l'archet y sette of Violes respectivamente ${ }^{21}$. El término viola se encuentra en las fuentes italianas desde finales del siglo XIII, incluso se halla en el Decameron de Boccaccio, y seguramente se refiere a un instrumento de cuerda pulsada apto para acompañar al canto22. Tanto la traducción francesa como inglesa, luc y lute, corroborarían esta hipótesis.

Obviamente, los términos viole da arco, vihuelas de arco, violles a l'archet y Violes nos remitirían a la familia de la viola da gamba. El cortesano ideal, pues, debía poder acompañarse al canto por un instrumento de cuerda pulsada o de mano y ser capaz de interpretar polifonía con uno de arco. Sabemos que el propio Castiglione era un excelente cantante que se acompañaba alla viola²3.

En la tratadística cinquecentesca existen más referencias a los instrumentos propicios para el moderno gentiluomo. Alessandro Piccolomini habla de la viola, el laúd y el Gravicembali (seguramente se refiere a un instrumento de tecla capaz de interpretar polifonía) ${ }^{24}$. De la misma opinión es Annibale Guasco, que en 1586 advierte de la importancia no solo de practicar el canto diariamente con la ayuda de una "viola da gamba bene accordata" para poder leer música a libro, o sea, de una partitura, sino de aprender a tocar también el clavicémbalo y la viola entendida como instrumento de cuerda pulsada apto para el acompañamiento25. Aparte de la habilidad en el canto, el cortesano debía dominar el manejo de instrumentos suaves idóneos para asociarse con la voz y ligados a la intimidad de las estancias palaciegas, en contraposición a los instrumentos de mayor volumen, tales como las trompetas, chirimías y sacabuches propios de profesionales a sueldo de municipios, cortes e instituciones religiosas 26.

\footnotetext{
20 Stefano Lorenzetti, "Viola Da Mano e Viola Da Arco: Testimonianze Terminologiche Nel Cortegiano (1528) Di Baldassar Castiglione", ed. Renato Meucci, Liuteria Musica e Cultura, 1996, p. 4.

${ }^{21}$ Lorenzetti, Musica e identità nobiliare, p. 101.

22 Lorenzetti, "Viola Da Mano e Viola Da Arco: Testimonianze Terminologiche Nel Cortegiano (1528) Di Baldassar Castiglione", p. 6.

${ }^{23}$ Lorenzetti, p. 20.

24 Alessandro Piccolomini, Della istitution morale, Libri XII, (Venecia: Francesco Ziletti, 1583). Citado por Stefano Lorenzetti, "Musica Vis in Animum. On the Relationship between Vocal and Instrumental Practice in the Pedagogical Treatises of the XVIth Century," en Le Concert Des Voix et Des Instruments à La Renaissance, ed. Jean-Michel Vaccaro, (Paris: CNRS, 1995), p. 43.

25 "[...] ti potrai nel canto e nel suono tutto a un tempo essercitare, e ti gioverà, come ti giovò di qui indietro, cotale strumento pure assai nel canto. Et oltre alla viola. Ottima compagnia ti farà il clavicordo, strumento molto più della viola compiuto; col quale potrai nelle sopradette cose da te stessa nel canto aiudarti". Annibale Guasco, Ragionamento ad Lavinia sua figliuola della maniera di governarsi in corte (Turín: the heir to Bevilacqua, 1586), p. 20. Citado por Lorenzetti, "Musica vis in animum. On the relationship between vocal and instrumental practice in the pedagogical treatises of the XVIth century", p. 46.

${ }^{26}$ Las fuentes tardo-medievales y renacentistas distinguen entre dos tipos de instrumentos. Por un lado los altos o haut, básicamente instrumentos de viento (excepto los de la familia de las flautas de pico), propios de profesionales que solían poder tocar igualmente instrumentos de cuerda aptos para la danza
} 


\section{El príncipe como músico y patrón}

El complejo mundo de los centros de poder del Renacimiento italiano podía dibujarse a modo de recreación a escala de las antiguas polis clásicas. La corte como círculo central de un sistema de aros concéntricos a modo de ondas que se expanden hacia el exterior abarcando toda la sociedad. Y, en el núcleo, la figura del príncipe ${ }^{27}$.

La imagen del príncipe como elemento central de autoridad se moldeó a nivel político en una evolución que arranca en la Italia comunal de los siglos XII y XIII a través de un proceso de patrimonialización del poder por parte de familias y clanes con suficiente apoyo para desembocar en tiranías gobernadas por signori que pasaron a controlar las fuentes recaudatorias y limitar la potestad de los consejos municipales. Con las tiranías y las señorías disminuyó la autoridad de los gobiernos comunales y republicanos mientras se afianzaba el dominio aristocrático. A pesar de ello, muchas de las antiguas familias patricias urbanas sobrevivieron y entraron a formar parte de las redes clientelares de los nuevos señores, de quienes recibieron toda clase de privilegios ${ }^{28}$. En ocasiones el acceso al poder implicó auténticos golpes de estado29. Los tiranos procedieron a legitimar su autoridad para mantener a la descendencia en el cargo. Es sintomático el caso de Giangaleazzo Visconti para conseguir el título de duque de Milán por parte del emperador a finales del siglo XIV30, y cómo una vez obtenido demostró la inteligencia suficiente para tolerar cierto autogobierno por parte de las ciudades del estado31. A pesar de todo, los procesos vividos en Italia desde la baja edad media hasta el siglo XVI no fueron homogéneos, ya que mientras el Milán de los Visconti, los Sforza, la dominación francesa y el gobierno de Felipe II permitió el desarrollo de un fuerte sistema de patriciado que controló la administración de justicia y las instituciones administrativas, el reino aragonés de Nápoles conservó un marcado acento feudal en su forma de gobierno incluso en el

como los violines y con una función social relacionada con lo festivo y las grandes celebraciones cívicas; y por otro los suaves o bas, tales como órganos, clavicémbalos, laudes, flautas de pico, arpas o violas da gamba, idóneos para las acústicas de palacio y el acompañamiento de la voz. En este segundo caso encontramos tanto a músicos profesionales como a cortesanos y a los propios monarcas. Victor Coelho y Keith Polk, Instrumentalist and Renaissance Culture, 1420 -1600: Players of Function and Fantasy, (Cambridge: Cambridge University Press, 2019), pp. 5-7.

27 Elisa Prieto Conca, Castiglione y el arquetipo humanista en España: música y paideia, (Madrid: Devenir, 2017), p. 30.

28 P.J. Jones, "Communes and Despots: The City State in Late-Medieval Italy," en Communes and Despots in Medieval and Renaissance Italy, ed. John E. Law y Bernardette Patton, (Farnham: Ashgate, 2010), pp. 21-23.

${ }^{29}$ Es el caso de Paolo Guinigi, capitán y responsable de la defensa de la pequeña república de Lucca que accedió al poder en 1400 convirtiéndose en su señor. Paradójicamente, fue derrocado por otro golpe orquestado por ciudadanos descontentos en 1430. Christine Meek, "Whatever's Best Administered Is Best': Paolo Guinigi Signore of Lucca," en Communes and Despots in Medieval and Renaissance Italy, ed. John E. Law y Bernardette Patton, (Farnham: Ashgate, 2010), pp. 131-144.

${ }^{30}$ Giangaleazzo recibió la dignidad ducal del emperador Venceslao en 1395. Jane Black, "Giangaleazzo Visconti and the Ducal Title," en Communes and Despots in Medieval and Renaissance Italy, ed. John E. Law y Bernardette Patton, (Farnham: Ashgate, 2010), pp. 119-121.

${ }^{31}$ Black, p. 130. 
siglo XVI ${ }^{32}$. Paralelamente, algunas ciudades que no sufrieron el proceso de señorialización evolucionaron de un gobierno comunal a repúblicas oligárquicas en las que el poder recayó en un escaso grupo de ciudadanos ${ }^{33}$.

De tirano a señor, de señor a príncipe. Un príncipe que desde mediados del siglo XV será educado bajo el influjo del Humanismo que lo moldeará como al perfecto cortesano, y que reivindicará su condición guerrera mediante la exaltación del mundo caballeres $\mathrm{CO}^{34}$. El punto álgido de este proceso fue la publicación del Orlando Furioso de Ludovico Ariosto como elogio de la casa de Este, cuyo programa simbólico dotó de argumentos a buena parte de la producción cultural del siglo XVI hasta la difusión de los preceptos de Trento 35. A mediados del siglo XVI, el mundo del Orlando era la base del recitar poético, del teatro y hasta de la composición musical. Lo que inicialmente estaba destinado a las audiencias aristocráticas de Ferrara pasó a ser denominador común de la cultura europea36.

La imagen del príncipe y la corte que emerge en el siglo XVI, tras la llegada de españoles y franceses a Italia, estuvo rodeada de una gravitas no solo ética sino estética. La élite principesca impuso nuevas modas que rompieron con lo establecido en el siglo anterior. Se impuso el negro en todos sus matices. Las clases dominantes rompieron con la policromía multicolor de la representación cortesana del siglo XV visible en los frescos del palacio Schifanoia de Ferrara o en los de Mantegna del castillo de San Jorge de Mantua37.

Las cortes italianas se inspiraron, en un primer momento, en el modelo de Borgoña, lo cual facilitó el establecimiento de fuertes conexiones con ciudades de gran tradición artística y musical al norte de los Alpes ${ }^{38}$. Muchos prominentes músicos partieron hacia Italia durante la segunda parte del siglo XV buscando el patronazgo de sus príncipes ${ }^{39}$. A partir de 1470 fue habitual la contratación de cantantes e instrumentistas procedentes del norte de

\footnotetext{
32 Mozzarelli, Antico regime e modernità, pp. 21-30.

${ }^{33}$ Es el caso de Venecia, Florencia o Siena. Iain Fenlon, "Music and Society," en The Renaissance: From the 1470s to the End of the 16th Century, ed. Iain Fenlon, (Londres: The Macmillan Press, 1989), p. 23.

${ }^{34} \mathrm{El}$ encargo de los frescos artúricos del palacio ducal de Mantua a Pisanello por parte de Ludovico Gonzaga data de la década de los cuarenta del siglo XV. Fenlon, p. 25.

35 Durante la primera parte del siglo se produjo una auténtica fiebre de interpretaciones alegóricas del poema de Ariosto. Visto como una experiencia poética del neoplatonismo espiritual, el Orlando devino un vehículo de propagación de códices, estrategias culturales y modelos ideológicos entre las clases más cultas y nutrió de significado algunos de los programas artísticos del momento, como el bosque sagrado de Bomarzo o el nuevo palacio ducal de Parma. Roberto Venturelli, La corte farnesiana di Parma: 15601570: programmazione artistica e identità culturale, (Roma: Bulzoni, 1999), pp. 45-52.

${ }^{36}$ Laurie Stras, Women and Music in Sixteenth-Century Ferrara, (Cambridge: Cambridge University Press, 2018), pp. 148-149.

37 Amedeo Quondam, Tutti i colori del nero: moda e cultura del gentiluomo nel Rinascimento, (Costabissara: A. Colla, 2007), pp. 34-35. Más allá del ensayo de Quondam, la importancia del negro como color preferido por las élites europeas del siglo XVI ha sido motivo de reflexión en la exposición temporal organizada por el Museo Nacional del Prado entre el 25 de junio y el 29 de septiembre de 2019 dedicada a las similitudes entre la pintura española y de los Países Bajos en los siglos XVI y XVII: Wessel Krul et al., Velázquez, Rembrant, Vermeer. Miradas afines., ed. Alejandro Vergara, (Madrid: Museo Nacional del Prado, 2019), pp. 25-50.

${ }^{38}$ Es sintomático el caso de la corte de Felipe el Bueno y su interés por la música durante la primera parte del siglo XV. Coelho y Polk, Instrumentalist and Renaissance Culture, $1420-1600$, pp. 19-21.

${ }^{39}$ Fenlon, "Music and Society", pp. 24-26.
} 
Europa necesarios para el entretenimiento y el ceremonial en una suerte de expansión del modelo borgoñón ${ }^{40}$. Los músicos se dividían según el tipo de instrumento y su función: instrumentos altos o haut y suaves o bas ${ }^{4}$. Los instrumentos altos eran básicamente los de viento y conformaban bandas de trompetas y de piffari (agrupaciones de sacabuches o trombones y chirimías) mientras que los suaves eran grupos de instrumentos de arco, cuerdas pulsadas y tecla. Esta división se encontraba ya a finales del siglo XV en Milán, Mantua, Ferrara o Nápoles ${ }^{42}$. Cercanos a los príncipes y de origen italiano encontramos una serie de poetas-improvisadores cuya producción musical no ha llegado hasta nosotros al ser oral e improvisada ${ }^{43}$. Estos intérpretes gozaron de gran estima entre la aristocracia pues representaban el punto más álgido de la canción acompañada, tan valorada por Castiglione y las academias humanistas ${ }^{44}$. El caso italiano más conocido es el de Pietrobono, cantante y laudista miembro de la corte de Borso d'Este en Ferrara y pretendido en Mantua, Nápoles y Milán. Admirado por su arte en la declamación de poemas, se acompañaba así mismo con un instrumento de cuerda pulsada. Era conocido entre sus contemporáneos como Pietrobono del Chitarino ${ }^{45}$. Con una fama comparable a la de los grandes polifonistas de su tiempo como Josquin Desprez, fue apreciado por el teórico Johannes Tinctoris, que en su Liber de arte Contrapunti (1477) ya distinguió entre música escrita e improvisación ${ }^{46}$. Con todo, a pesar de que Pietrobono simbolizó un referente máximo a nivel musical del ideal cortesano, su trascendencia ha sido relativizada por la musicología, acostumbrada a otorgar poca importancia a la tradición no escrita, subordinada a la evanescencia propia de la ejecución.

Muchos de los príncipes de las más ilustres familias del norte de Italia mimaron de forma especial a la música, ejerciendo una labor de patronazgo intensa. A partir de la comisión de obras a compositores reputados a sueldo de la corte, el soberano procuraba una base permanente de intérpretes para ejecutar la música. Desde finales de la década de 1470 se encontraba en Milán un grupo estable de trompetas, de piffari, de tañedores de instrumentos de cuerda y tecla e incluso cantantes para la interpretación de la música profana, así como una capilla vocal responsable de la polifonía en los servicios religiosos (con un organista acompañante) ${ }^{47}$. Los cuatro grupos actuaban

\footnotetext{
40 Coelho y Polk, Instrumentalist and Renaissance Culture, p. 22.

${ }^{41}$ Ver nota 26. Coelho y Polk, pp. 5-7.

42 Fenlon, "Music and Society", p. 27.

${ }^{43}$ No solo los cantantes se basaban en la improvisación, algunos instrumentistas adquirieron gran fama sin depender de composiciones escritas, como el organista ciego al servicio del duque de Baviera Conrad Paumann o el dúo Fernández y Cordoval, laudistas e intérpretes de instrumentos de cuerda frotada, que en ocasiones actuaban a trío con un cantante. De todos ellos conocemos su existencia a través de las fuentes documentales. Coelho y Polk, Instrumentalist and Renaissance Culture, pp. 51-53.

${ }^{44}$ Ver nota 17.

45 Lewis Lockwood, Music in Renaissance Ferrara 1400-1505, (Oxford: Clarendon Press, 1984), p. 98.

46 Tinctoris establece dos tipos de contrapunto o modos de hacer música, el cantar super librum o improvisando, y la composición escrita o res facta. Coelho y Polk, Instrumentalist and Renaissance Culture, pp. 196-198.

47 William F. Prizer, "Music at the Court of the Sforza: The Birth and Death of a Musical Center", Musica Disciplina, 43, (1989), p. 143.
} 
separadamente. Los trompetistas eran de origen italiano y oscilaban entre cuatro y veinte. Su uso era preferentemente ceremonial. La mayor parte de los piffari, entre tres y seis a principios del siglo XVI, provenían de Alemania y amenizaban los torneos, procesiones y espectáculos de corte, aunque también colaboraban en las danzas de palacio. Los instrumentistas de cuerda o de música suave tocaban el laúd, la lira da braccio y posteriormente la viola da gamba. Podían acompañar a cantantes y actuaban en ambientes íntimos y restringidos. Su participación resultaba especialmente valorada al intervenir privadamente para los invitados del príncipe, hecho que servía para aumentar el prestigio del mismo. Eran utilizados también en la música de danza y en procesiones religiosas y podían estar a cargo de la instrucción musical de los hijos del soberano48. Al igual que los piffari, participaban en los intermedios de las comedias y en los banquetes ${ }^{49}$. Respecto a la capilla, en Milán oscilaba entre seis y trece cantantes a cargo de los servicios sacros, ya fuera en la corte o en la catedral50. Esta estructura se repitió, con matices, en las principales cortes. El momento de mayor esplendor musical de la capital lombarda correspondió con el reinado de Galeazzo Maria Sforza (1466-1477), hábil cantante cuya capilla fue la más extensa de su tiempo y que atrajo a compositores transalpinos tan eminentes como Alexander Agricola o Josquin Desprez ${ }^{51}$.

Las cortes en que las consortes demostraron una especial sensibilidad artística y musical llegaron a mantener diversas capillas, una para el príncipe y otra para la mujer. Los casos más célebres son los de Isabella d'Este (14741539) y Lucrezia Borgia (1480-1519), cuñadas y soberanas de Mantua y Ferrara respectivamente, cuya rivalidad en el patronazgo las convirtió en máximos ejemplos de donne di palazzo52. Lucrezia poseía un staff musical

\footnotetext{
48 Prizer, p. 144.

49 La importancia de los banquetes en la vida de la corte era máxima. De ellos dependía la reputación del príncipe, pues normalmente servían para agasajar a un invitado importante o para celebrar el enlace de un miembro de la familia gobernante con otro de una casa poderosa. La música resultaba fundamental dentro del protocolo del evento ayudando al ritmo y entretenimiento durante los servicios. Resulta especialmente interesante la descripción de una cena ofrecida en Ferrara por Ercole d'Este, duque de Chartres, a su padre, el duque Alfonso I, el 24 de enero de 1529. Tras la representación de la comedia La Cassaria de Ariosto, los invitados pasaron a otra estancia junto a una estufa para escuchar música esperando que la gran mesa estuviera preparada. El primer servicio entró anunciado por la trompetería y mientras los comensales disfrutaban del mismo cantó la célebre Madonna Dalida con otras cuatro voces, así como el cantante Alfonso Santo con cinco violas da gamba y un clavicémbalo. En el segundo servicio participaron cuatro cantantes interpretando madrigales variados. En el tercero se ejecutó un diálogo a ocho voces partido en dos coros mixtos de voces e instrumentos. En el cuarto música compuesta por el maestro de capilla Alfonso della Viola para cinco cantantes, cinco violas, una especie de contrabajo y una dulzaina. Para el quinto cinco trombones y un corneto, en el sexto actuaron dos mujeres cantantes y cinco varones, entre ellos Ruzzante (1502-1541), apodo de Angelo Beodo, célebre actor y dramaturgo en dialecto pavano. El banquete terminó con la intervención de bufones alrededor de la mesa en el séptimo plato y de los piffari en el octavo y último servicio. Todo el proceso de la cena lo detalla Cristoforo de Messisbugo, administrador ducal de la corte estense, en su tratado de cocina de 1549: Cristoforo Messisbugo, «Dai "Banchetti, composizioni di vivande et apparecchio generale" (1549)», en L'arte della cucina in Italia, ed. Emilio Faccioli, (Turín: Einaudi, 1987), pp. 298-304.

50 Prizer, "Music at the Court of the Sforza", p. 145.

51 Prizer, pp. 152-159.

${ }^{52}$ Concepto de ideal cortesano ya anunciado por Castiglione y reservado a las mujeres de más alta alcurnia que debían dominar el arte de la conversación, la danza, el canto y la pericia en el tañer ciertos instrumentos. Federico Schneider, "An Amused Muse: The donna di palazzo and Music in the Cortegiano", L'analisi linguistica e letteraria, 10, 1-2, (2002), p. 450.
} 
formado por frottolistas, cantantes, músicos de cuerda y un profesor de danza, y era de menores dimensiones que el de su marido, el duque Alfonso d'Este, ya que no contaba con trompetas, piffari y un coro de música sacra ${ }^{53}$. En caso de necesidad, podía contratarlos de las capillas del duque o de su cuñado el cardenal Ippolito54. La capilla privada de Isabella era semejante a la de Lucrezia con el añadido de un instrumentista de tecla. Al igual que su cuñada, podía contar con cantantes para el repertorio sacro o grupos de viento provenientes de la capilla de su marido, Francesco II Gonzaga, marqués de Mantua55.

El duque Alfonso I d'Este, representaba el prototipo de príncipe amante de la música. Al igual que su hermana tocaba la viola da gamba durante las primeras décadas de desarrollo del instrumento. Por una carta del constructor afincado en Venecia Lorenzo da Pavia a Isabella sabemos que "el señor Don Alfonso [d'Este] se encuentra en Venecia y desea que le haga cinco 'viole da archo', y con la mayor insistencia. No ayuda decir que yo no puedo hacerlas. Él desea que las haga de todos los tamaños del mundo. Su señoría desea aprender" 56 .

El propio Alfonso tocó en un consort de seis viole durante las celebraciones de su boda con Lucrezia en 1502, lo cual indica que su dominio del instrumento era notable ${ }^{57}$. Isabella había empezado a aprender a tocar la viola un poco antes. En una carta a su hermanastro Giulio del 14 de mayo de 1499 se alude a que "hemos empezado desde el principio a aprender la viola y esperamos que la aprendamos bien, porque en los dos días en los que hemos estado trabajando, hemos empezado a hacer planes de modo que, cuando vengamos a Ferrara, podremos tocar el tenor con Don Alfonso"58.

Isabella estaba casada con un Gonzaga, familia que accedió al poder de Mantua en 1328 en la llamada Cacciata dei Bonacolsi, que expulsó a los Bonacolsi de la ciudad y situó a los Gonzaga como capitanes del popolo y vicarios imperiales. En un primer momento su dominio señorial fue relativo hasta el capitán Francesco I, que en torno 1400 tomó el control del podestà

\footnotetext{
${ }^{53}$ Lucrezia disponía de un profesor de danza, disciplina de gran importancia en el marco cortesano definido por Castiglione. Este aspecto de la cortesanía ha sido estudiado por especialistas en la materia como Diana Campóo Schelotto, "La danza y el lenguaje de la virtud en El Cortesano de Baldassare Castiglione", Annali di Storia Moderna e Contemporanea, Nuova Serie, Anno II, (2014), pp.9-30.

54 William F. Prizer, "Isabella d'Este and Lucrezia Borgia as Patrons of Music: The Frottola at Mantua and Ferrara", Journal of the American Musicological Society, 38, 1, (1985), pp. 12-14.

55 William F. Prizer, "Renaissance Women as Patrons of Music: The North-Italian Courts", en Institutions and Patronage in Renaissance Music, ed. Thomas Schmidt-Beste, (Ashgate, Farnham, 2012), pp. 176177.

56 "L'è e qua a Venecia il Signore Don Alfonso, al quale vole li faci 5 viole da archo, e con grandissima instancia. Non me vale a dire che io non lo posso fare, e'l vole a tuti i modi del mondo che li face. La sua Signoria vole imparare". I-MAas, (Archivio di Stato, Mantua), Busta 1438, fol. 614. Transcrita en Prizer, "Isabella d'Este and Lorenzo Da Pavia, 'Master Instrument-Maker"', p. 105 .

57 "[...] una música de sei viole, fra quale vi era il Signor don Alfonso [...]". Ian Woodfield, The Early History of the Vio, I, (Cambridge: Cambridge University Press, 1984), p. 89.

58 "Havemo ben dato principio ad imparare de viola et speramo che impareremo assai bene perchè in dui dì solamente che gli havemo dato opera, cominciamo a fare dele minute per modo che, quando veniremo a Ferrara, potremo fare tenore alo Illustrissimo Signor Don Alphonso nostro fratello". I-MAas, Busta 2993, Libro 10, fol. 15v. Transcrita en Prizer, "Isabella d'Este and Lorenzo Da Pavia, 'Master Instrument-Maker'", p. 105.
} 
o juez supremo y del massaro u oficial financiero. Con el hijo de Francesco, Gianfrancesco, el dominio se volvió más tiránico y se eliminaron dos de los tres consejos comunales, aunque la familia siempre tuvo la habilidad de dejar cierta autonomía a la oligarquía urbana. En 1433 el capitán Gianfrancesco pasó a ser marqués consolidando el poder de la dinastía59. Una de las características de los Gonzaga fue la estrecha relación entre el tronco principal y las ramas menores de la familia (Guastalla, Bozzolo, Sabbioneta, Luzzara y los pueblos de Castelgoffredo, Castiglione y Solferino, cada uno controlado por un clan menor), hecho que animó a la circulación de artistas, músicos y escritores. En Sabbioneta, Vespasiano Gonzaga construyó un teatro siguiendo el modelo clásico de la Accademia Olimpica de Vicenza. Otro miembro de una rama colateral, Ferrante Gonzaga (1507-1557), conde del recién estado independiente de Guastalla desde 1539, hospedó siendo niño al compositor Orlando di Lasso y mantuvo contactos con Nicolás Gombert60.

El momento culminante de su carrera se produjo al ser nombrado gobernador de Milán en 1546 tras la muerte del marqués del Vasto61. Su hijo Ferrante, condottiere como el padre, luchó en Flandes y en el Mediterráneo contra los piratas, fundó la Accademia degli Invaghiti instalada en el palacio familiar de la ciudad y patrocinó la publicación de diversas colecciones de música, algunas de ellas del polifonista Giaches de Wert.62

Figura especialmente destacada de la familia fue la del cardenal Ercole (1505-1563), hijo del duque Francesco e Isabella d'Este. Como segundo vástago de nueve fue educado para la carrera eclesiástica según los patrones humanistas. La muerte de su hermano Federico le convirtió en corregente del ducado en 1540 junto a Ferrante. Figura clave en el desarrollo del concilio de Trento, presidió la tercera sesión desde noviembre de 1561 hasta su muerte el 2 de marzo de 156363. El compromiso con la Contrarreforma guio buena parte de la política de mecenazgo del cardenal. Encargó a Giulio Romano, que había trabajado para el hermano Federico en el Palazzo del Te, la reforma de la catedral dotándola de una renovada vida musical, pasando a tener un corpus de 18 cantantes en 1528 a 32 en 1565. Para dirigir la capilla contrató a Jacquet de Mantua, que era pagado por el propio prelado. Como obispo de Mantua mantuvo una corte privada en el palacio episcopal64.

Ercole es el paradigma de príncipe y cortesano dentro de la Iglesia, una muestra que los ideales humanistas se integraron plenamente en la curia

\footnotetext{
59 David S. Chambers, "The Gonzaga Signoria, Communal Institutions and the 'the Honour of the City': Mixed Ideas in Quattrocento Mantua", en Communes and Despots in Medieval and Renaissance Italy, ed. John E. Law y Bernardette Patton, (Farham: Ashgate, 2010), pp. 106-115.

${ }^{60}$ Fenlon, Music and Patronage in Sixteenth-Century Mantua. Vol. 1, pp. 31-32.

${ }^{61}$ Antonio Álvarez-Ossorio Alvariño, Milán y el legado de Felipe II: gobernadores y corte provincial en la Lombardía de los Austrias, (Madrid: Sociedad Estatal para la Conmemoración de los Centenarios de Felipe II y Carlos V, 2001), pp. 45-64.

62 Fenlon, Music and Patronage in Sixteenth-Century Mantua. Vol. 1, pp. 32-33.

63 Fenlon, p. 55.

${ }^{64}$ Se han conservado registros de pagos a cantantes e instrumentistas, incluso a instrumentistas de viento y tambores del palacio ducal que ocasionalmente trabajaron para el cardenal. Fenlon, pp. 64-65.
} 
romana65. En la literatura dedicada a la cortesanía, tras la obra de Castiglione se encuentra el De Cardinalatu de Paolo Cortese, publicado en 1510 y dedicado a Julio II. Cortese trata de fijar el código de comportamiento del cardenal-príncipe mediante una suerte de totum revolutum de preceptos, consejos y avisos sobre el funcionamiento de la corte, sus miembros y aparato66. El segundo libro de la obra, el Liber oeconomicus, reflexiona sobre el rol del cardenal en la vida pública como príncipe de la Iglesia diferenciándolo muy poco del tipo de corte que rodea a un gobernante secular. Cortese abogaba por un extravagante nivel de vida: la famiglia estaría formada por 140 miembros y se necesitarían 12.000 ducados anuales para costear los gastos. La corte residiría en un gran palacio siguiendo las más recientes teorías arquitectónicas que albergaría un cubiculum musicae, una de las primeras referencias a un espacio exclusivamente musical67.

La idea del cubiculum musicae sugiere la idoneidad de un espacio dedicado ex profeso a la música en palacio. En Italia la vida de corte posibilitó la existencia de salones y estancias pensadas para la ejecución musical68. Existe una carta de Claudio Monteverdi que describe un evento musical acaecido en la sala de los espejos del palacio ducal de Mantua que puede considerarse un testimonio no solo de una forma de actuar sino la narración de una modalidad de recepción, de la transformación de un ambiente en un "quasi novo teatro" pensado para la interpretación ${ }^{69}$.

En ocasiones la existencia alrededor del príncipe podía resultar tan compleja que un mismo edificio llegaba a albergar varias cortes, a modo de submundos o satélites, que orbitaban en torno al monarca. En Ferrara, durante la década de 1580, la vida musical cortesana ligada a los actos más protocolarios como banquetes, recepciones, festejos para el carnaval o celebraciones religiosas dependía de los músicos del duque Alfonso II, que se agrupaban en una gran formación llamada concerto grande ${ }^{70}$. Algunos de sus miembros en ocasiones participaban en la llamada musica secreta, una suerte

\footnotetext{
65 Fenlon, "Music and Society", p. 15.

${ }^{66}$ Quondam, El discurso cortesano, p. 38.

67 Fenlon, "Music and Society", p. 15.

68 Stefano Lorenzetti, "Musica Nello Specchio Della Corte. Qualche Riflessione Su Appartenenza e Presenza", en Le Parole Che Noi Usiamo. Categorie Storiografiche e Interpretative Dell'Europa Moderna, ed. Marcello Fantoni y Amedeo Quondam, (Roma: Bulzoni, 2008), p. 192.

69 "Ogni veneri di sera si fa musica nella Sala de'Specchi. Viene a cantare in concerto la signora Andriana, e così fatta forza particular grazia dà alle composizioni, aportando così fatto diletto al senso, che quasi novo teatro divien quel loco; e creddo che non si finirà il carnevale de'concerti, che sarà di bisogno che il serenissimo signor Duca facci stare guardia al'entrata, ché giuro a Vostra Signoria Illustrissima che questo venere passato, ad udire, non solamente il serenissimo signor Ducca e serenissima signora Duchessa, la signora donna Isabella di Santo Martino, signor Marchese e Marchesa di Solfarino, signore damme e cavaglieri di tutta la corte vi erano, ma più de cento signori altri de la città ancora. Con tal bella occasione farò sonare li chitaroni a li casalaschi nel'organo di legno, il quale è soavissimo, e cosí canterà la signora Adriana e don Giovanni Battista il madregale bellisimo 'Ahi che morire mi sento' e I'altro madregale ne'l organo solamente. Dimani porterò le dette composizioni, presentandole alla signora Andriana, e so quanto le saranno care; né voglio dirle il nome del'autore sino a tanto che ella non le averà cantate, e de la riuscita de tutto ne darò raguaglio Vostra Signora Illustrissima". Mantua, 22 de enero de 1611. Carta de Claudio Monteverdi al cardenal Ferdinando Gonzaga de Roma. Claudio Monteverdi, Lettere, ed. Éva Lax, (Florencia: L. S. Olschki, 1994), pp. 33-34.

70 Anthony Newcomb, The madrigal at Ferrara, 1579-1597, Princeton studies in music, 7, (Princeton, N.J.: Princeton University Press, 1980), p. 33.
} 
de veladas reservadas a los invitados más especiales y protagonizadas por el concerto delle dame, mujeres cantantes del séquito de la duquesa Margherita Gonzaga, esposa de Alfonsoı.

El concerto solía actuar en las habitaciones de Margherita, pero paralelamente al mismo, su cuñada Lucrezia d'Este, duquesa de Urbino, que residía en el mismo castello, formó su propio concerto, que se reunía de forma similar en sus apartamentos privados ${ }^{72}$. Dos grupos de exclusivas cortesanascantantes conviviendo paralelamente en diferentes espacios del mismo palacio, dos pequeñas cortes, la de la duquesa de Ferrara y la duquesa de Urbino ${ }^{73}$.

Entre 1580 y 1582 las interpretaciones de la musica secreta se alternaron en las estancias de las dos señoras en un proceso de progresivo enfriamiento de su relación, ya que la llegada de Margherita a Ferrara en 1579 para casarse con Alfonso relegó a Lucrezia a un segundo plano ${ }^{74}$.

\section{Mujer y corte: donne di palazzo, cortesanas y profesionales}

La literatura renacentista ha legado a la posteridad un considerable número de retratos femeninos dedicados a mujeres virtuosas. En un afán ejemplarizante, no son pocos los tratados que consideraban al sexo femenino igual al masculino. Este tópico no era nuevo y tenía sus raíces en la Edad Media75.

Mientras la pedagogía humanista abrió a la mujer las puertas del conocimiento, la literatura de corte reformó la imagen de esta. Castiglione nos recuerda que cada placer y diversión que sucede en la corte se hace para agradar a la dama, convertida en fuente dispensadora de placer. Aun así, algunas de las maneras y comportamientos propios de la mujer no difieren sustancialmente de la de los hombres, tal como se describe en el tercer capítulo del segundo libro:

"Quiero que esta Dama tenga noticia de letras, de música, de pinturas, y sepa danzar bien, y traer, como es razón, a los que andan con ella de amores, acompañando siempre con una discreta templanza, y con

\footnotetext{
${ }^{71}$ El concerto delle dame ha sido motivo de un especial interés por parte de la historiografía de la música por su carácter enigmático y su innegable importancia en la evolución del madrigal y el desarrollo del virtuosismo vocal. Entre las obras más importantes dedicadas al tema podemos citar la de Newcomb, The madrigal at Ferrara, 1579-1597., Elio Durante y Anna Martellotti, "Giovinetta Peregrina": La Vera Storia Di Laura Peperara e Torquato Tasso, (Florencia: L. S. Olschki, 2010)., o a Stras, Women and Music in Sixteenth-Century Ferrara.

72 Elio Durante y Anna Martellotti, Cronistoria Del Concerto Delle Dame Principalissime Di Margherita Gonzaga d'Este, (Florencia: Spes, 1989), pp. 58-59.

${ }^{73} \mathrm{El}$ modelo del concerto de la duquesa Margherita proporcionó a la corte de Alfonso un prestigio enorme y fue imitado en Florencia, Roma y Mantua. Newcomb, The madrigal at Ferrara, 1579-1597, pp. 90-101. 74 Newcomb, p. 101.

75 Entre los tratados humanistas que reivindican la condición femenina podemos citar De laudobis mulieribus de Bortolomeo Gogio, Defensio mulierum de Agostino Strozzi y De mulieribus de Mario Equicola, seguramente impreso a principios del siglo XVI, que incluye entre una galería de celebridades femeninas un intrigante retrato de Isabella d'Este como músico. Stefano Lorenzetti, "Public behavior, music and the construction of feminine identity in the Italian Renaissance", Recercare, 23, 1-2, (2011), pp. 7-8.
} 
dar buena opinión de sí, todas aquellas otras consideraciones que han sido enseñadas al Cortesano; y haciéndolo así, parecerá bien a todos hablando o riendo, en juegos, en burlas, y, en fin, en cuanto hiciere, y sabrá entretener discretamente y con gusto a cuantos tratare" 76 .

El ejercicio de la gracia definirá las acciones de la mujer en palacio. Una gracia que definió Annibale Romei en sus Discorsi como «una cierta facilidad y agilidad que tiene el cuerpo al obedecer el alma»77. En virtud de ella, la neoplatónica separación entre mente y cuerpo se fusiona configurando un ideal de belleza cimentada en la perfección del cuerpo y la excelencia del espíritu:

"Pues yo, respondió el Manífico, tengo licencia de formar esta Dama a mi placer, no solamente no quiero que use esos exercicios tan impropios para ella, pero quiero que aun aquellos que le convienen los trate mansamente, y con aquella delicadeza blanda que, según ya hemos dicho, le pertenece. Y así en el danzar no querría vella con unos movimientos muy vivos y levantados, ni en el cantar o tañer me parecería bien que usase aquellas disminuciones fuertes y replicadas que traen más arte que dulzura; asimismo los instrumentos de música que ella tañiere estoy en que sean conformes a esta intinción; imaginá agora cuan desgraciada cosa sería ver una mujer tañendo un atambor, o un pífano, o otros semejantes instrumentos; y la causa desto es la aspereza dellos, que encubre o quita aquella suavidad mansa que propiamente y bien se asienta en las mujeres. Por eso, y si alguna vez le dixeren que dance o taña o cante, debe esperar primero que se lo rueguen un poco, y cuando lo hiciere, hágalo con un cierto miedo"78.

Más allá de los instrumentos aptos para el sexo femenino, en el que los vientos y las percusiones quedan excluidos como ya sucedía en las recomendaciones al gentiluomo, Castiglione aconsejaba a la dama un modo de entender la música alejado de la artificiosidad y el virtuosismo ("disminuciones fuertes y replicadas"). El virtuosismo como tal estará reservado a las profesionales. El concepto de sprezzatura vuelve a aparecer con la idea de que la ejecución musical debe huir de la artificiosidad, disimulando "il studio e la fatica" propia de la práctica79.

El ideal de mujer humanista que propone la tratadística como encarnación de un ser excelente y virtuoso puede ejemplarizarse en la figura desgraciada

\footnotetext{
${ }^{76}$ Castiglione, El cortesano, p. 314.

77 "Una certà facilità e agilità che ha il corpo ad ubidir all'anima". Angelo Solerti y Annibale Romei, Ferrara E La Corte Estense: Del Secolo Decimosesto: I Discorsi Di Annibale Romei, (Breslavia: ULAN Press, 2012), p. 14.

78 Castiglione, El cortesano, p. 312.

${ }^{79}$ Lorenzetti, "Musica vis in animum. On the relationship between vocal and instrumental practice in the pedagogical treatises of the XVIth century", p. 43.
} 
de Irene di Spilimbergo, muerta en 1559 a los 21 años. Su desaparición provocó tal desazón entre sus contemporáneos que en 1561 Dionigi Atanagi publicó un libro con 229 poemas en latín y lengua vulgar escritos por algunos de los intelectuales más importantes de su tiempo80. Nacida el 17 de octubre de 1539 en Spilimbergo, al norte de Udine, era hija de un noble filoprotestante fallecido en 1541. Irene fue enviada a Venecia a los 15 años a vivir con sus abuelos maternos donde adquirió fama como música y pintora. Según Atanagi, siempre mostró signos de una inteligencia extraordinaria8ı. Estudió música con el laudista Bartolomeo Gazza y con Tromboncino82. A partir de la descripción que aporta Atanagi en el volumen conmemorativo queda claro que en su tiempo Irene ya fue admirada como el prototipo de mujer según el modelo de Castiglione: interesada en lo intelectual pero no pedante, cómoda en compañía de hombres de letras y artistas, brillante pero no prepotente, eterna aspirante a la perfección en todos sus quehaceres, en especial la pintura83.

A pesar de la visión idealizada de la mujer en la corte, la cotidianidad en palacio proporcionaba multitud de oportunidades de mostrar a la princesa como amante de las artes, en especial la música, tanto en su aspecto de ejecutante o intérprete como en su labor de patrocinio. La necesidad del estudio y la práctica, tanto literaria como musical, provocó que en ocasiones se reservaran estancias donde la intimidad y el sosiego permitieran el aislamiento imprescindible para el cultivo de las artes. Isabella d'Este, siguiendo el ejemplo de Federico de Montefeltro, se hizo construir un studiolo en el palacio ducal de Mantua decorado con motivos musicales ${ }^{84}$. En el ciclo de siete pinturas encargadas para este espacio puede observarse el interés de la marquesa por la dicotomía entre instrumentos de cuerda y de viento ${ }^{85}$.

${ }^{80}$ Algunos de los literatos que participaron en el homenaje fueron Scipione Ammirato, Giuseppe Betussi, Lodovico Dolce, Lodovico Domenichi, Girolamo Muzio, Bernardo y Torquato Tasso o el propio Tiziano Vecellio. El índice del volumen publicado por Dionigi Atanagi en Venecia, Rime di diversi nobilissimi et eccellentissimi autori in morte della signora Irene delle signore di Spilimbergo (1561), ha sido editado como facsímil. Anne Jacobson Schutte, "Commemorators of Irene di Spilimbergo", Renaissance Quarterly, 45, 3, (1992), pp. 524-536.

${ }^{81}$ Anne Jacobson Schutte, "Irene di Spilimbergo: The Image of a Creative Woman in Late Renaissance Italy", Renaissance Quarterly, 44, 1, (1991), pp. 49-50.

82 Hasta hace poco tiempo se creía que Irene estudió canto con Bartolomeo Tromboncino, uno de los más importantes autores e intérpretes de frottole, que estuvo al servicio de Isabella d'Este y Lucrezia Borgia. Sin embargo, recientes investigaciones apuntan a que fue el hijo de Bartolomeo, Ippolito, su verdadero maestro. Paolo Da Col, "Silent Voices: Professional Singers in Venice", en A Companion to Music in Sixteenth-Century Venice, ed. Katelijne Schiltz, (Leiden: Brill, 2018), p. 269.

${ }_{83}$ Arte en el que sobresalió, llegando a aprender en el taller de Tiziano, que no solía aceptar alumnos. En solo seis semanas, según Atanagi, copiando las obras del maestro, llegó a tener un dominio excelso de la técnica de la pintura. Schutte, "Irene di Spilimbergo", pp. 51-53.

${ }^{84}$ Lorenzetti, Musica e identità nobiliare nell'Italia del rinascimento, pp. 178-79.

85 Con Isabella representada a sí misma como amore celeste, o como la Venus del amor espiritual, uno de los temas expuestos es la oposición entre instrumentos de viento y de cuerda. En la primera pintura, completada por Andrea Mantegna en 1497, Apolo con su lira y Mercurio con sus flautas de pan están representados opuestamente. En la pintura de Lorenzo Costa Comos, la Venus del amor físico, sostiene también una flauta de pan, e intenta prevenir a Apolo, con su lira, de unirse a la Venus del amor espiritual, mientras Amphion, el mítico poeta-músico, tañe una lira da braccio y se dirige al rescate de Janus y Mercurio, ocupados en combatir a los Vices. En la pintura de Costa La Coronación, una noble, seguramente la propia Isabella, es coronada reina del jardín mientras al fondo se representa una batalla acompañada por instrumentos de viento. Finalmente, la Alegoría de la Virtud y Vice de Correggio, muestran a la Virtud con una lira y a Vice con una flauta. Isabella participó activamente en el diseño de la temática de las 
Isabella se consideraba a sí misma como el arquetipo de mujer amante de la música, que no ocultaba su interés por aprender continuamente, pero siempre sujeta a la retórica de la modestia. Se presentó a la boda de su hermano Alfonso con Lucrezia Borgia con varios laúdes y ataviada con un vestido bordado repleto de representaciones simbólicas de claves musicales, signos de silencio y mensurales. A pesar de esta imagen tan musical, no cantó en el banquete hasta que los cortesanos le suplicaron que lo hiciera86. Su apego a la música no se conformaba con poseer una capilla privada. Se ha comentado que estaba en contacto con el constructor Lorenzo da Pavia, al cual no solo le encargaba instrumentos, sino que actuaba como su agente en Venecia para conseguir joyas, piezas de orfebrería y otras rarezas87. Algunos de los instrumentos que encargaba la marquesa a Lorenzo no solo se requerían para ser tocados por ella, sino que eran utilizados por sus músicos profesionales. 88

El papel de Isabella en el desarrollo de la viola da gamba en los primeros años de vida del instrumento es evidente. Tanto su hermano como ella aprendieron a tocar la viola, al menos, desde 149989. Un poco antes, en 1495, ordenó cuatro instrumentos a un anónimo constructor de Brescia90. Se trata del primer encargo de violas da gamba conocido. En marzo de 1495 Isabella se encontraba en Brescia y ordenó a su agente Marco Nigro, la compra de tres viole. Nigro era el prefecto de las municiones, pero no sabía de música. Los instrumentos eran referidos como viole over lire ${ }^{91}$. Según documentación posterior parece que no eran ni lire da braccio ni fídulas, por lo que seguramente se trataba de violas da gamba de diferentes tamaños conocidas en Italia al menos desde la interpretación en Vigevano, cerca de Milán, de un concierto de viole de diferentes tamaños por parte de músicos españoles venidos de Roma en 149392. En agosto de 1495, Isabella añadía al encargo una viola grande presumiblemente para interpretar la voz de bajo en la polifonía93. En la correspondencia que trata esta compra se observa el interés

\footnotetext{
pinturas del studiolo, indicando a los artistas los asuntos a representar. Prizer, "Isabella d'Este and Lorenzo Da Pavia, 'Master Instrument-Maker'", pp. 115-116.

${ }^{86}$ Stras, Women and Music, pp. 84-85.

87 Lorenzo Gusnasco nació en Pavía alrededor de 1470 o 1475. Antes de 1494 abrió una tienda en Venecia que permaneció activa hasta 1515 , teniendo entre sus clientes no solo a Isabella sino a la cuñada Lucrezia Borgia, a los Este de Ferrara, los Sforza de Milán o los Montefeltro y della Rovere de Urbino. Incluso el papa León X requirió de sus servicios. Prizer, "Isabella d'Este and Lorenzo Da Pavia, 'Master InstrumentMaker"', p. 88.

${ }^{88}$ Prizer, p. 117.

89 Ver nota 58.

90 Woodfield, The Early History of the Viol, p. 94.

${ }^{91}$ Carta de Nigro a Isabella, 7 de mayo de 1495. I-MAas, Busta 1599. Transcrita en Prizer, "Isabella d'Este and Lorenzo Da Pavia, 'Master Instrument-Maker'", p. 102.

92 La interpretación formaba parte de las celebraciones del nacimiento de Massimiliano Sforza, heredero de Ludovico el Moro. Algunas de las violas eran tan grandes como una persona, tal como el canciller de Ferrara Bernardino de'Prosperi, testigo del evento, relata a Isabella en una carta del 6 de marzo de 1493. Prizer, p. 101.

93 Carta de Isabella a Nigro, 22 de agosto de 1495. I-MAas, Busta 2993, Libro 10, fol. 41r. Transcrita en Prizer, p. 103.
} 
de Isabella por obtener el mejor precio posible. Su determinación y habilidad para abaratar el coste inicial está bien documentada94.

La marquesa de Mantua, paradigma de donna di palazzo, velando por los gastos particulares con el mismo celo que cualquier mujer con la economía familiar. El microcosmos de la corte interactuando de forma directa y ordinaria con agentes, artesanos, constructores y todo tipo de elementos del mundo urbano circundante. La relación de príncipes y princesas con cortesanos y elementos ajenos a su rango era tan cotidiana como compleja.

Las mujeres de la casa de Este también mostraron un gran interés por la música a lo largo del siglo XVI. Leonora (1515-1575), la única hija de Alfonso I y Lucrezia, nació cuatro años antes de la muerte de su madre e hizo un uso muy diferente de su educación musical. Formalmente ingresó en el convento de Corpus Domini con tan solo ocho años y allí residió hasta su muerte, convirtiéndose en su abadesa95. La vida de Leonora en el convento resultó confortable y privilegiada, con permiso para mantener contacto privadamente con elementos externos. Interesada a nivel práctico y teórico por la música, poseía diversos instrumentos de teclado. El maestro de capilla del duque, Francesco dalla Viola, le dedicó su Primo libro de madrigali a quattro voci9. Lo que llama la atención de la princesa monja es que se dedicó activamente a la composición, publicando durante la década de 1540 diversos motetes escritos para voci pari97.

La ausencia de Leonora de la vida pública provocó un extraño vacío femenino en la corte hasta la llegada con 18 años de la hija de Luis XII de Francia, Renée de Valois, para casarse con el hermano de Leonora, el futuro Ercole II. Renée se estableció en el palacio de San Francesco, cercano al castello, donde mantuvo una pequeña capilla musical de origen francés. Su vida en Ferrara estuvo marcada por sus creencias protestantes, que provocaron no pocas contrariedades al ducado98. Las dos hijas de Renée y Ercole, Anna (nacida en 1531), que se casó con el duque de Guisa, y Lucrezia (nacida en 1535), futura duquesa de Urbino, no siguieron las ideas

\footnotetext{
94 En una misiva de Marco Nigro a Isabella, del 27 de junio de 1495 el agente informa de que "Zuan Angelo, sonator de Vostra Sublimità, vene qui e conpite le viole. Insieme con lui fu cum maistro per concluder el mercato, qual maistro, che ha del bizaro, me rechiexe XXV ducati. Zuan Angelo el menò via e disse che'l conzeria la cossa. Tandem eri el maistro vene a mi, dicendo che el voleva li danari et io li offersi a darli, pur fossamo d'acordo del precio, el qual lui par de non contentarse de le XV ducati li offersi. Zuan Angelo voleva li desse XVIII [...]". I-MAas, Busta 1599. Tres días más tarde, Isabella envió a un mulatero a recoger las violas con 15 ducados con instrucciones de cancelar el encargo en caso de no aceptar el constructor el precio: "Mandamovi per il cavalaro presente quindece ducati per scodere quelle nostre viole. Quando el maestro se ne contenti potete dargele. Quando anche el volesse più dinari, chè le non valeno, poteti dirli che'l ne disponi al piacere suo, chè nui non le volemo [...]". Carta de Isabella d'Este a Marco Nigro, 30 de junio de 1495. I-MAas, Busta 2992, Libro 5, fol. 49r. Transcritas en Prizer, p. 125.

95 Stras, Women and Music, p. 64.

96 Venecia: Gardano, 1550.

97 Voces de registro agudo, lo que convertía estas obras en aptas para ser cantadas por las monjas. Stras, Women and Music, pp. 36-37.

98 Stras, pp. 66-67.
} 
reformistas de su madre y recibieron una educación propia a su rango, distinguiéndose por su destreza en el canto99.

Las cortes más refinadas vieron cómo a lo largo del siglo aparecieron una serie de mujeres con gran capacidad musical, con fama de cantar, danzar y tocar instrumentos en el que el elemento virtuosístico era considerado digno de admirar. A medida que avanza el siglo XVI la bravura vocal se integró de forma más evidente en la ilusión de la cortesanía. Muchas de estas mujeres no eran consideradas profesionales como tales, a veces pertenecían a la aristocracia local y en otras ocasiones se les proporcionaba un matrimonio que implicara el acceso a la pequeña nobleza ${ }^{100}$. En Ferrara, durante el período de gobierno del heredero de Renée y Ercole, Alfonso II, se produjo una importante presencia de estas cortesanas para integrarse en el concerto delle dame de la musica secreta.

Tras el terremoto que asoló Ferrara en 1570 Alfonso trasladó la corte a la población cercana de Brescello. El 31 de julio de 1571 llegaron allí las hermanas Lucrezia e Isabella Bendidio, que anteriormente habían servido a las princesas Anna y Lucrezia pero que ahora formaban parte del cortejo de la duquesa Barbara. Ambas adquirieron fama como cantantes y actuaban tanto juntas como separadas. Lucrezia Bendidio, amante del hermano del duque, el cardenal Luigi d'Este, provocaba una especial admiración por su forma de cantar. El poeta Giambattista Pigna relata en un soneto su estilo de canto, repleto de ornamentos y gruppetti de gran virtuosismo. 101

En 1576 se produjo la llegada a la corte de Alfonso II del cantante bajo Giulio Cesare Brancaccio y la noble de origen parmesano Leonora Sanvitale. 102 Ambos destacaban en el nuevo estilo de canto basado en la bravura y en un

\footnotetext{
99 La diferencia entre la educación de una mujer de clase media y una princesa estaba pensada para que la primera pudiera ser capaz de velar por la vida doméstica de una casa y la segunda supiese administrar el gobierno del estado en caso de ausencia del marido, que en ocasiones estaba ocupado en asuntos militares. Stras, p. 70.

${ }^{100}$ La existencia de este tipo de cortesanas se confunde con el concepto de cortigiana onesta, meretrices de elevada cultura y aptitudes musicales que trasladaban algunas de las virtudes de la elitista donna di palazzo a la esfera pública. El caso más conocido fue el de la veneciana Veronica Franco. Shawn Marie Keener, "Virtue, Illusion, Venezianità: Vocal Bravura and the Early Cortigiana Onesta", en Musical Voices of Early Modern Women: Many-Headed Melodies, ed. Thomasin Lamary, (Routledge, Londres, 2017), pp. 119-120. La cortigiana onesta recreaba pequeñas cortes en sus salones, frecuentados por intelectuales, hombres de estado, eclesiásticos o mercaderes. Su eclosión significó un ennoblecimiento del oficio de la prostitución. Lorenzetti, Musica e identità nobiliare nell'Italia del rinascimento, p. 147. En 1537 llegó a Ferrara la cortesana romana Tullia d'Aragona, hija de una meretriz y del cardenal Luigi d'Aragona (15101556), estableció su propio salón y adquirió un status equivalente al de la más alta noble virtuosa. En una carta a Isabella d'Este, el informante Servitor Apollo la describe como educada, discreta, inteligente y poseedora de divinas maneras. No es juzgada de forma maliciosa sino admirativamente. De ella se resalta su capacidad para leer en partitura cualquier canción o motete. Stras, Women and Music, p. 60.

101 "In giri or lunghi, or scarsi, or doppi, or soli/ or alti, or bassi, netta voce sgorga:/ e con silenzio e strepito la ingorga/ il vostro augel, perché a me morte involi". Il ben divino, CXX. Pigna reunió los poemas dedicados a Lucrezia en un volumen manuscrito, Il ben divino. Citado por Stras, Women and Music, p. 172.

102 Leonora estuvo ligada a la familia Farnese. Nacida en 1558 o 1559, era la hija de Giberto Sanvitale, conde de Sala, funcionario de la famiglia del papa Pablo III en Roma. Giberto enviudó y se casó con Barbara Sanseverina, no mucho mayor que la hija. La primera referencia de Leonora con el canto se conserva en una carta de su madrastra al cardenal Alessandro Farnese escrita en agosto de 1573, en la que pedía al prelado permiso para cambiar de profesor de canto. Más tarde, gracias a la intervención del hermano del cardenal, el duque Ottavio de Parma, Leonora se casó con el conde de Scandiano a finales de 1575, pocos días antes de partir para Ferrara. Stras, pp. 184-190.
} 
efectismo exagerado que estaba triunfando en Roma. La canción a solo volvía a estar de moda en detrimento de la polifonía, pero sin el sosiego melódico de la frottola. ${ }^{103}$ Brancaccio sería utilizado como intérprete de la voz más grave del concerto delle dame.

Las cantantes que dominaron la escena de la corte estense en la década de 1570, las hermanas Bendidio y Leonora Sanvitale, pertenecían a la nobleza y no eran consideradas profesionales. Simplemente habían cultivado de forma excepcional sus habilidades musicales. Eran admiradas por la elegancia de su voz y sus maneras en la danza. Estaban integradas en el círculo de la hermana de Alfonso, la duquesa de Urbino, que había retornado a Ferrara tras un matrimonio desastroso con Francesco Maria Della Rovere104. El paradigma cambió a partir de 1579 con la boda del duque con Margherita Gonzaga y la formación del concerto delle dame105.

La llegada de Margherita significó la formación del concerto, que actuaba de forma reservada en las estancias de la nueva soberana. Las integrantes del grupo, Laura Peperara, Anna Guarini y Livia d'Arco, habían obtenido un elevado rango social gracias a sus habilidades musicales y luego se casaron con nobles para asegurar su posición en la corte106. Laura Peperara era de origen mantuano, tocaba el arpa y era admirada por su belleza. Su fama era tal que la Accademia dei Filarmonici de Verona le dedicó un libro de madrigales tras una visita a la ciudad con el cortejo del duque Guglielmo Gonzaga de Mantua en 1578, antes de su partida para Ferrara 107. Una vez en la capital estense, Alfonso organizó sus nupcias con el conde Annibale Turco para integrarla en la aristocracia local108. La boda, celebrada el 22 de febrero de 1583, fue solemnizada con festejos que incluyeron un balletto ideado por la duquesa Margherita, consumada bailarina. Poetas y músicos le dedicaron una colección de madrigales titulado Il Lauro Verde ${ }^{109}$. El año anterior ya había sido protagonista de un primer libro de homenajel10. Entre los poetas que escribieron los textos estaba Torcuato Tasso, que había conocido a Laura de niña en Mantua. El literato, que había dedicado un cancionero amoroso a

\footnotetext{
103 En su Discorso sopra la musica, Vincenzo Giustiniani situa el año de 1575 como el inicio de esta nueva forma de canto. Giustiniani comenta que la voz de Brancaccio tenía una extensión de 22 notas (casi tres octavas) y era capaz de abarcar desde el registro más grave al más agudo mediante pasajes de gran bravura. Vincenzo Giustiniani, Discorso Sopra La Musica (1628), trad. Carol MacClintock, Musicological Studies and Documents, 9, (Roma: American Institute of Musicology, 1962), p. 69.

${ }_{104}$ Lucrezia era quince años mayor que el novio, heredero del ducado de Urbino. Se casaron en enero de 1570. La vida conyugal resultó un desastre y tras el ascenso de Francesco Maria al trono del ducado en 1574 decidió volver a Ferrara. Stras, Women and Music, p. 182.

105 Newcomb, The madrigal at Ferrara, 1579-1597, p. 10.

106 Stras, Women and Music, p. 218.

107 En Verona las aptitudes musicales de Laura cautivaron a los miembros de la academia. Durante y Martellotti, Giovinetta peregrina, pp. 84-85.

108 Durante y Martellotti, pp. 209-215.

109 Durante y Martellotti, Cronistoria del concerto delle dame principalissime di Margherita Gonzaga d'Este, p. 29.

${ }_{110}$ Il Lauro Secco, publicado en 1582 por el impresor ducal Baldini. Durante y Martellotti, Giovinetta peregrina, p. 216.
} 
Lucrezia Bendidio en 1561, compuso multitud de poemas para la Peperara en la década de 1580 '1!.

A pesar de la tensa relación de Tasso con Alfonso, que llegó a encerrarlo durante siete años por un desgraciado accidente en el castello ante las damas de Margherita en la boda de los duques, puede considerarse la figura del poeta tan necesaria en la corte de Ferrara como la del propio príncipe, ya que ejercía la función de establecer por escrito los valores del estado'12.

La segunda de las cantantes del concerto secreto fue Anna Guarini, sobrina de las hermanas Bendidio. Si Laura podía acompañarse con el arpa, Anna era una excelente laudista।13. Las dos damas solían actuar con el acompañamiento al bajo de Giulio Cesare Brancaccio, tal como lo atestigua una colección de seis madrigales de Tasso de 1581 o $1582^{\prime 14}$.

La tercera de las componentes del grupo era Livia d'Arco, que llegó a Ferrara con quince años en el séquito de Margherita. Estudió viola da gamba con el organista de la corte Luzzascho Luzzaschi, que solía acompañar a las damas con el clavicémbalo115. Una vez finalizó su instrucción musical se integró en el grupo a finales de 1582. Las tres mujeres pasaron a formar parte inseparable del cortejo de la duquesa, habitando en el castello y acompañando a su señora en los paseos en carrozal16. En la corte recibieron la tutela musical de Giaches de Wert, maestro de capilla ducal desde 1565 y uno de los polifonistas más reputados de su tiempo, y de Luzzaschi, responsable musical del concerto ${ }^{117}$.

Entre los afortunados huéspedes que tenían acceso a las exclusivas veladas de la musica secreta en los aposentos de la duquesa destacan la de los músicos florentinos Giovanni Bardi y Giulio Caccini, que jugaron un papel esencial en la invención de la óperalı8. Su experiencia en los conciertos de las damas convenció al Gran Duque de Toscana a enviar al compositor Alessandro Striggio con tal de que compusiera madrigales inspirados en la forma de cantar del concerto, en una suerte de espionaje artístico. Fruto de ello escribió un diálogo para dos sopranos con ornamentaciones muy vistosas con acompañamiento pensado para Caccini. Lo que más impresionó al visitante fue que las señoras cantaban de memoria:

\footnotetext{
${ }^{111}$ Conocidas como Rime per Laura Peperara, están catalogados en Durante y Martellotti, pp. 327-333.

112 La necesaria relación de Tasso con Alfonso II tiene su paralelo en la función de poetas como Garcilaso respecto a Carlos V o Francisco de Aldana con Felipe II. Prieto Conca, Castiglione y el arquetipo humanista en España, p. 30.

113 Stras, Women and Music, p. 219.

${ }_{114}$ Recopilados en las Rime del poeta. Durante y Martellotti, Cronistoria Del Concerto, p. 243.

115 Durante y Martellotti, p. 29.

116 Stras, Women and Music, p. 220.

117 Durante y Martellotti, Cronistoria Del Concerto, p. 27.

118 La florentina Camerata Bardi, de la cual formaba parte también Vincenzo Galilei, padre de Galileo Galilei, es considerada capital en el cambio estético que sufre la música a principios del siglo XVII. Enrico Fubini, La Estética Musical Desde La Antigüedad Hasta El Siglo XX, trad. Carlos Guillermo Pérez de Aranda (Madrid: Alianza Editorial, 1988), pp. 141-143.
} 
"[...] El señor Duque me favorece continuadamente mostrándome por escrito todas las obras que cantan de memoria, con todas las tiradas y pasajes que hacen, y espero que en ocho o diez días su Alteza se contentará que yo retorne a Mantua, donde he dejado a mi mujer e hijos, y allí podré cómodamente, a imitación de estos cantos de Ferrara, componer alguno para el concerto de V.A. [...]"II9

A las tres damas habría que añadir como animadora de la vida musical ferrarense a Tarquinia Molza, originaria de Módena, que había inspirado a Alfonso en la creación de un grupo femenino de voces tras una velada en su ciudad natal en el remoto otoño de $1568^{120}$. Tras enviudar en 1579 y visitar Ferrara diversas veces, se trasladó a la corte en 1583. Allí protagonizó una escandalosa relación amorosa con Giaches de Wert que provocó su caída en desgracia. En 1577 el filósofo Francesco Patrizi compuso el diálogo inacabado L'amorosa filosofia, dedicado a Molza, en el que glosaba admirativamente el arte de Tarquinia, en especial su pericia a la hora de acompañarse con la viola da gamba y cantar la melodía al mismo tiempo'21.

La boda de Margherita con Alfonso significó un eclipse en el protagonismo de la duquesa de Urbino en la corte, hecho que la empujó a recluirse en sus estancias y formar un concerto alternativo con cuatro miembros, Vittoria Guarini, hermana de Laura, y las hermanas Giulia, Lucrezia y Ginevra Avogadri, que actuaban acompañadas al órgano por Vicenzo Bonizzi ${ }^{122}$.

Todo acabó con la muerte del Alfonso en 1597. Los concertos se disolvieron, Margherita retornó a Mantua el mismo año para dedicarse a la vida religiosa hasta su deceso en 1618, mientras que la duquesa de Urbino falleció en 1598 sin poder impedir que la ciudad pasase a manos de los territorios pontificios.

\footnotetext{
119 "[...] Il sig. Duca mi favorisse di continuo di mostrarmi in scritto tutte le opere che cantano a la mente, con tutte le tirate e passaggi che vi fanno, e spero che fra otto o dieci giorni sua Altezza si contentarà ch'io ritorni a Mantova, dove ho lassato mia moglie e figli, e là io potrò cómodamente, ad imitatione di questi canti di Ferrara comporne qualche uno per il concerto di V.A. [...]". Carta del 29 de julio de 1584 de Alessandro Striggio a Francesco I, Gran Duque de Toscana. Transcrita en Durante y Martellotti, Cronistoria Del Concerto, p. 165.

120 Durante y Martellotti, Giovinetta Peregrina, p. 241.

121 Su capacidad para ornamentar con pasajes de bravura mientras se acompañaba era inaudita, superando a afamados músicos como Alfonso Ferrabosco: "[...] E questi è poi stato dalla signora Tarquinia di lungo superato. Con ciò sia che il Ferabosco nelle dificultà e ne'passi stretti ove l'occhio non può supplire al bisogno di vedere tutte le note una ad una, ei ricorre al contrapunto e riempie que'vacui che I'occhio converrebbe di lasciare non tocche. Ma la signora obligandosi a tutte le note ad una ad una per minime o semiminime che sieno, et a tutte le parole, supera anco questa difficultà si grande, con grande stupore di chiunge la vede a ciò fare et ode. [...]". Texto de Francesco Patrizi del diálogo L'amorosa filosofia, de 1577. Transcrito en Durante y Martellotti, Cronistoria Del Concerto, p. 136.

122 Durante y Martellotti, Giovinetta Peregrina, pp. 238-239. Bonizzi publicó una serie de glosas para viola bastarda en 1626 dedicadas a Giulia Avogadri, que aparte de cantar en el grupo de la duquesa de Urbino era una virtuosa de la viola da gamba: Vicenzo Bonizzi, Alcune Opere, Di Diversi Auttori a Diverse Voci, Passaggiate Principalmente per La Viola Bastarda, Ma Anco per Ogni Sorte Di Stromenti, e Di Voci, (Venecia: Alessandro Vincenti, 1626), facsímil editado por Elio Durante y Anna Martellotti, Archivium Musicum, 54, (Florencia: Spes, 1983).
} 


\section{El azar y la fortuna, paradigmas de la experiencia cortesana}

A pesar de la ilusión poética que desprendía la vida de corte, la imprevisibilidad y la fragilidad gobernaban la condición del cortesano con respecto a los príncipes. Su posición social dependía de la relación con el soberano y podía caer en desgracia en cualquier momento. Lucrezia Bendidio fue aislada una vez su amante, el cardenal Luigi, partió hacia Francia en labores diplomáticas en 1573. Las calumnias emborronaron su figura hasta el punto de que Alfonso prohibió al secretario y a la duquesa que hablaran con ella. El ostracismo a que fue sometida no la expulsó del castello, pero su rastro desapareció de los listados de dame de la corte ${ }^{123}$. Tarquinia Molza fue desterrada de la ciudad con su amante Giaches de Wert en septiembre de 1589. A pesar de la viudedad de ambos, la unión fue considerada inapropiada por los duques por su avanzada edad ${ }^{24}$. La fortuna de Anna Guarini tras la muerte de Alfonso resultó calamitosa. Víctima de la difamación, se la acusó falsamente de infidelidad en 1596. El duque se erigió en su protector, pero a su muerte fue cruelmente asesinada por el marido con un hacha, con la complicidad del hermano de la desdichada, el 3 de mayo de 1598125. Quien corrió mejor fortuna fue Laura Peperara, que heredó a la muerte de la duquesa de Urbino un valioso instrumento'26.

El elemento vocal masculino del grupo, el bajo Giulio Cesare Brancaccio, representa el paradigma de guerrero-cortesano del siglo XVI. Perteneciente a la pequeña nobleza napolitana, participó en multitud de conflictos bélicos llegando a ser gentilhomme ordinaire de la chambre du roi en la corte de Enrique II de Francia ${ }^{27}$. Llegó a Ferrara como cantante afamado rondando los sesenta años. Orgulloso de su pasado como soldado, estaba obsesionado con publicar unos comentarios sobre la obra de Julio César. Un primer momento de desencuentro en la corte se debió a un comportamiento inapropiado en la residencia ducal de Belriguardo, por su insistencia en departir sobre temas militares y su reticencia a cantar. Este hecho le alejó del ducado en 1579 . Al suplicar su regreso, Alfonso le puso como condición no conversar de guerra y participar en la musica secreta128. El viejo napolitano volvió a Ferrara en diciembre de 1580 para ser expulsado definitivamente en julio de 1583 al negarse a cantar en el concerto e insistir en discutir sobre el arte bélico ${ }^{129}$.

\footnotetext{
123 Stras, Women and Music, pp. 176-77.

124 Molza fue expulsada del servicio de la duquesa Margherita y retornó a Módena, continuando con su actividad musical hasta su muerte en 1617 . Wert volvió a Mantua, donde previamente había prestado servicio. Stras, p. 295.

125 Stras, p. 312.

126 Durante y Martellotti, Cronistoria Del Concerto, p. 201.

127 Richard Wistreich, Warrior, courtier, singer: Giulio Cesare Brancaccio and the performance of identity in the late Renaissance, (Abingdon: Ashgate, 2007), p. 52.

128 En una carta del 31 de octubre de 1580 al Gran Duque de Toscana Francesco el embajador florentino en Ferrara Urbani le informa de que: "[...] aspetta fra brevi giorni al suo servitio il Sig. Giulio Cesare Brancaccio, ma che ha fatto patto con lui che non habbia a parlare di quei suoi miracoli di guerra, ma sì bene intervenir talvolta in una musica secreta che si va preparando d'alcune Dame della corte le quali tuttavia attendono a farsi studio [...]". Transcrita en Durante y Martellotti, Cronistoria Del Concerto, p. 138.

${ }^{129}$ Durante y Martellotti, p. 34. También en Wistreich, Warrior, courtier, singer, pp. 272-273.
} 
Brancaccio se refugió en Venecia, pero pronto se estableció en Padua bajo el patronazgo de Vittoria Accoramboni, esposa de Paolo Giordano Orsini, duque de Bracciano. Parece ser que el anciano y excéntrico guerrero murió asesinado junto a su protectora en un novelesco desenlace en diciembre de $1585^{130}$.

A pesar del final abrupto del concerto y de muchos de sus miembros, su legado ha sido de tal importancia que años después Vincenzo Giustiniani comentaba de esta forma su innovadora manera de entender el arte de la interpretación:

"[...] entre ellas las señoras de Mantua y Ferrara, que hacían gala, no solo en cuanto al metal y disposición de las voces, sino en el ornamento de exquisitos pasajes ejecutados en oportuna conjura y no soberbia (en la que solía pecar Gio. Lucca falsetista de Roma, que sirvió incluso en Ferrara), y más en moderar y crecer la voz forte y piano, debilitándola o engrandeciéndola, que según venía cortada, ahora arrastrándola, ahora partiéndola con el acompañamiento de un suave suspiro, ahora cantando pasajes largos, bien encadenados, articulados, ahora grupos, ahora saltos, ahora con trinos largos, ahora con cortos, y con pasajes suaves cantados piano, de manera que de pronto se sentían ecos responder, principalmente con acciones del rostro, y de miradas y de gestos que acompañaban apropiadamente la música y los significados, $[\ldots]^{\prime \prime} \mid 31$

\footnotetext{
130 La historia fue novelada en el siglo XIX por Domenico Gnoli. A causa de desavenencias en el reparto de la herencia del reciente fallecido duque de Bracciano, el hermano del finado envió unos sicarios al palacio de Vittoria el 22 de diciembre durante un evento musical, asestando una puñalada en la garganta de Brancaccio y matando a la princesa en su propia cama. Wistreich, Warrior, courtier, singer, p. 124. 131 "[...] fra quelle dame di Mantova et di Ferrara, che facevano a gara, non solo quanto al metallo et alla disposizioni delle voci, ma nell'ornamento di esquisiti passaggi tirati in opportuna congiuntura e non soverchi, (nel che soleva peccare Gio. Luca falsetto di Roma, che servì anche in Ferrara), e di più col moderare e crescere la voce forte o piano, assottigliandola o ingrossandola, che secondo che veniva a'tagli, ora con strascinarla, ora smezzarla, con l'accompagnamento d'un soave interrotto sospiro, ora tirando passaggi lunghi, seguite bene, spicatti, ora gruppi, ora a salti, ora con trilli lunghi, ora con brevi, et or con passaggi soavi e cantati piano, dalli quali talvolta all'improviso si sentiva echi rispondere, e principalmente con azione del viso, e dei sguardi e de'gesti che acompagnavano appropriatamente la musica e i concetti,[...]". Vincenzo Giustiniani, Discorso sopra la musica, 1628. Transcrito en Durante y Martellotti, Cronistoria Del Concerto Delle, p. 153.
} 
Bibliografía:

Álvarez-Ossorio Alvariño 2001: Antonio Álvarez-Ossorio Alvariño, Milán y el legado de Felipe II: gobernadores y corte provincial en la Lombardía de los Austrias, (Madrid: Sociedad Estatal para la Conmemoración de los Centenarios de Felipe II y Carlos V, 2001).

Black 2010: Jane Black, "Giangaleazzo Visconti and the Ducal Title", en Communes and Despots in Medieval and Renaissance Italy, editado por John E. Law y Bernardette Patton, (Farham: Ashgate, 2010), pp.119-130.

Bonizzi 1626: Vicenzo Bonizzi, Alcune opere, di diversi auttori a diverse voci, passaggiate principalmente per la viola bastarda, ma anco per ogni sorte di stromenti, e di voci, (Venecia: Alessandro Vincenti, 1626), facsímil editado por Elio Durante y Anna Martellotti, Archivium Musicum, 54, (Florencia: Spes, 1983).

Campóo Schelotto 2014: Diana Campóo Schelotto, "La danza y el lenguaje de la virtud en El Cortesano de Baldassare Castiglione", Annali di Storia Moderna e Contemporanea, Nuova Serie, Anno II, (2014), pp.9-30.

Castiglione 2008: Baldassare Castiglione, El cortesano, traducido por Juan Boscán, (Madrid: Alianza Editorial, 2008).

Castiglione 1981: Baldassare Castiglione, Il Libro del Cortegiano con una scelta delle Opere minori, editado por Bruno Maier, (Turín: UTET, 1981).

Chambers 2010: David S. Chambers, "The Gonzaga Signoria, Communal Institutions and the "the Honour of the City": Mixed Ideas in Quattrocento Mantua», en Communes and Despots in Medieval and Renaissance Italy, editado por John E. Law y Bernardette Patton, (Ashgate, Farnham, 2010), pp. 105-118.

Coelho y Polk 2019: Victor Coelho y Keith Polk, Instrumentalist and Renaissance Culture, 1420 -1600: Players of Function and Fantasy, (Cambridge: Cambridge University Press, 2019).

Da Col 2018: Paolo Da Col, "Silent Voices: Professional Singers in Venice", en A Companion to Music in Sixteenth-Century Venice, editado por Katelijne Schiltz, (Leiden: Brill, 2018), pp. 230-271.

Durante y Martellotti 1989: Elio Durante y Anna Martellotti, Cronistoria del concerto delle dame principalissime di Margherita Gonzaga d'Este, (Florencia: Spes, 1989).

Durante y Martellotti 2010: Elio Durante y Anna Martellotti, "Giovinetta peregrin"»: la vera storia di Laura Peperara e Torquato Tasso, (Florencia: L. S. Olschki, 2010).

Fenlon 1980: Iain Fenlon, Music and Patronage in Sixteenth-Century Mantua. 
Volume 1, (Cambridge: Cambridge University Press, 1980).

Fenlon 1989: Iain Fenlon, "Music and Society", en The Renaissance: from the 1470 s to the end of the 16th century, editado por Iain Fenlon, (Londres: The Macmillan Press, 1989), pp.1-62.

Fubini 1988: EnricoFubini, La estética musical desde la Antigüedad hasta el siglo XX, traducido por Carlos Guillermo Pérez de Aranda, (Madrid: Alianza Editorial, 1988).

Giustiniani 1962: Vincenzo Giustiniani, Discorso sopra la musica (1628), traducido por Carol MacClintock, Musicological Studies and Documents, 9, (Roma: American Institute of Musicology, 1962).

Guasco 1586: Annibale Guasco, Ragionamento ad Lavinia sua figliuola della maniera di governarsi in corte, (Turín: the heir to Bevilacqua, 1586).

Guazzo 1577: Stefano Guazzo, La civil conversatione, (Venecia: Salicato, 1577).

Hoffmann 2004: Bettina Hoffmann, "Del concerto alto al concerto basso: accordature delle viole da gamba nell'Italia del Cinquecento", Recercare, 16, (2004), pp. 23-67.

Jones 2010: P.J. Jones, "Communes and Despots: The City State in LateMedieval Italy", en Communes and Despots in Medieval and Renaissance Italy, editado por John E. Law y Bernardette Patton, (Farnham: Ashgate, 2010), pp. 3-26.

Keener 2017: Shawn Marie Keener, "Virtue, Illusion, Venezianità: Vocal Bravura and the Early Cortigiana Onesta", en Musical Voices of Early Modern Women: Many-Headed Melodies, editado por Thomasin Lamary, Londres: (Routledge, 2017), pp. 119-133.

Krul, Portús, Posada Kubissa, Van de Pol y Vergara 2019: Wessel Krul, Javier Portús, Teresa Posada Kubissa, Barber Van de Pol, y Alejandro Vergara, Velázquez, Rembrant, Vermeer. Miradas afines, editado por Alejandro Vergara, (Madrid: Museo Nacional del Prado, 2019).

Lockwood 1984: Lewis Lockwood, Music in Renaissance Ferrara 1400-1505, (Oxford: Clarendon Press, 1984).

Lorenzetti 1995: Stefano Lorenzetti, "Musica vis in animum. On the relationship between vocal and instrumental practice in the pedagogical treatises of the XVIth century", en Le Concert des voix et des instruments à la Renaissance, editado por Jean-Michel Vaccaro, (París: CNRS, 1995), pp.3950.

Lorenzetti 1996: Stefano Lorenzetti, "Viola Da Mano e Viola Da Arco: Testimonianze Terminologiche Nel Cortegiano (1528) Di Baldassar Castiglione", editado por Renato Meucci, Liuteria Musica e Cultura, (1996), pp. 2-23. 
Lorenzetti 1997: Stefano Lorenzetti, «"Per animare agli esercizi nobili". Esperienza musicale e identità nobiliare nei collegi di educazione», Quaderni Storici 32, 2, (1997), pp. 435-460.

Lorenzetti 2003: Stefano Lorenzetti, Musica e identità nobiliare nell'Italia del rinascimento: educazione, mentalità, immaginario, (Florencia: L.S. Olschki, 2003).

Lorenzetti 2008: Stefano Lorenzetti, "Musica nello specchio della corte. Qualche riflessione su appartenenza e presenza", en Le parole che noi usiamo. Categorie storiografiche e interpretative dell'Europa moderna, editado por Marcello Fantoni y Amedeo Quondam, (Roma: Bulzoni, 2008), pp. 187-196.

Lorenzetti 2011: Stefano Lorenzetti, "Public behavior, music and the construction of feminine identity in the Italian Renaissance", Recercare, 23, 1-2, (2011), pp. 7-34.

Mariani 2014: Jacob A. Mariani, "From Poet's aid to Courtier's pastime: an Examination of the Shift in visual style and sounding function of Italian Viols during the Renaissance", Degree of Master of Arts, University of Oregon, 2014.

Meek 2010: Christine Meek, "Whatever's Best Administered is Best': Paolo Guinigi signore of Lucca", en Communes and Despots in Medieval and Renaissance Italy, editado por John E. Law y Bernardette Patton, (Farnham: Ashgate, 2010), pp.131-144.

Messisbugo 1987: Cristoforo Messisbugo, «Dai "Banchetti, composizioni di vivande et apparecchio generale" (1549)», en L'arte della cucina in Italia, editado por Emilio Faccioli, (Turín: Einaudi, 1987), pp.284-326.

Monteverdi 1994: Claudio Monteverdi, Lettere, editado por Éva Lax, (Florencia: L. S. Olschki, 1994).

Mozzarelli 2008: Cesare Mozzarelli, Antico regime e modernità, (Roma: Bulzoni, 2008).

Newcomb 1980: Anthony Newcomb, The madrigal at Ferrara, 1579-1597, 2 vols., Princeton studies in music, 7, (Princeton, N.J.: Princeton University Press, 1980).

Nosow 2002: Robert Nosow, "The Debate on Song in the Accademia Fiorentina", Early Music History, 21, (2002), pp. 175-221.

Piccolomini 1583: Alessandro Piccolomini, Della istitution morale Libri XII, (Venecia: Francesco Ziletti, 1583).

Prieto Conca 2017: Elisa Prieto Conca, Castiglione y el arquetipo humanista en España: música y paideia, (Madrid: Devenir, 2017).

Prizer 1982: William F. Prizer, "Isabella d'Este and Lorenzo Da Pavia, 'Master Instrument-Maker'", Early Music History, 2, (1982), pp. 87-127. 
Prizer 1985: William F. Prizer, "Isabella d'Este and Lucrezia Borgia as Patrons of Music: The Frottola at Mantua and Ferrara", Journal of the American Musicological Society, 38, 1, (1985), pp. 1-33.

Prizer 1989: William F. Prizer, "Music at the Court of the Sforza: The Birth and Death of a Musical Center", Musica Disciplina, 43, (1989), pp. 141-93.

Prizer 2011: William F. Prizer, "Performance Practices in the Frottola", Early Music, 3, 3, (2011), pp. 227-235.

Prizer 2012: William F. Prizer, "Renaissance Women as Patrons of Music: The North-Italian Courts", en Institutions and Patronage in Renaissance Music, editado por Thomas Schmidt-Beste, (Farnham: Ashgate, 2012), pp. 161-190.

Quondam 2007: Amedeo Quondam, Tutti i colori del nero: moda e cultura del gentiluomo nel Rinascimento, (Costabissara: A. Colla, 2007).

Quondam 2013: Amedeo Quondam, El discurso cortesano, editado por Eduardo Torres Corominas, (Madrid: Polifemo, 2013).

Reese 1995: Gustav Reese, La música en el Renacimiento, traducido por José María Martín Triana, 2 vols., (Madrid: Alianza Editorial, 1995).

Schneider 2002: Federico Schneider, "An Amused Muse: The donna di palazzo and Music in the Cortegiano", L'analisi linguistica e letteraria, 10, 1-2, (2002), pp. 449-460.

Schutte 1991: Anne Jacobson Schutte, "Irene di Spilimbergo: The Image of a Creative Woman in Late Renaissance Italy", Renaissance Quarterly, 44, 1 (1991), pp. 42-61.

Schutte 1992: Anne Jacobson Schutte, "Commemorators of Irene di Spilimbergo", Renaissance Quarterly, 45, 3, (1992), pp. 524-36.

Solerti y Romei 2012: Angelo Solerti y Annibale Romei, Ferrara E La Corte Estense: Del Secolo Decimosesto: I Discorsi Di Annibale Romei, (Breslavia: ULAN Press, 2012).

Stras 2018: Laurie Stras, Women and Music in Sixteenth-Century Ferrara, (Cambridge: Cambridge University Press, 2018).

Venturelli 1999: Roberto Venturelli, La corte farnesiana di Parma: 15601570: programmazione artistica e identità culturale, (Roma: Bulzoni, 1999).

Wistreich 2007: Richard Wistreich, Warrior, courtier, singer: Giulio Cesare Brancaccio and the performance of identity in the late Renaissance, (Abingdon: Ashgate, 2007).

Woodfield 1984: Ian Woodfield, The Early History of the Viol, (Cambridge: Cambridge University Press, 1984). 